\title{
High-energy constraints from low-energy neutrino nonstandard interactions
}

\author{
Jorge Terol-Calvo ${ }^{1,{ }^{1 *}}$ Mariam Tórtola $\odot,{ }^{1,2, \dagger}$ and Avelino Vicente $\oplus^{1, \$}$ \\ ${ }^{1}$ Instituto de Física Corpuscular (CSIC-Universitat de València), C/ Catedrático José Beltrán 2, \\ E-46980 Paterna (València), Spain \\ ${ }^{2}$ Departament de Física Teòrica, Universitat de València, 46100 Burjassot, Spain
}

(Received 7 January 2020; accepted 20 April 2020; published 12 May 2020)

\begin{abstract}
Many scenarios of new physics predict the existence of neutrino nonstandard interactions, new vector contact interactions between neutrinos, and first generation fermions beyond the Standard Model. We obtain model-independent constraints on the Standard Model effective field theory at high energies from bounds on neutrino nonstandard interactions derived at low energies. Our analysis explores a large set of new physics scenarios and includes full one-loop running effects below and above the electroweak scale. Our results show that neutrino nonstandard interactions already push the scale of new physics beyond the $\mathrm{TeV}$. We also conclude that bounds derived by other experimental probes, in particular by low-energy precision measurements and by charged lepton flavor violation searches, are generally more stringent. Our study constitutes a first step toward the systematization of phenomenological analyses to evaluate the impact of neutrino nonstandard interactions for new physics scenarios at high energies.
\end{abstract}

DOI: 10.1103/PhysRevD.101.095010

\section{INTRODUCTION}

The Standard Model (SM) provides a successful description of a vast amount of particle physics phenomena. This includes many predictions at high-energy colliders, with the recent discovery of the Higgs boson as the latest example, as well as an astonishing agreement with a long list of precision measurements performed at low-energy experiments. However, despite its success, there are several well-known problems the SM cannot address. Among them, the existence of nonzero neutrino masses is arguably the most robust one. After the discovery of neutrino flavor oscillations, it has become clear that the leptonic sector of the SM must be extended with some additional states responsible for the generation of neutrino masses. Even though the underlying physics is not known, this fact has been completely established due to the high precision achieved in the determination of the neutrino oscillation parameters [1].

Many neutrino mass models have been proposed over the years. In most scenarios, the new Beyond the Standard Model (BSM) degrees of freedom have masses well above

\footnotetext{
*jorge.terol@ific.uv.es

mariam@ific.uv.es

*avelino.vicente@ific.uv.es
}

Published by the American Physical Society under the terms of the Creative Commons Attribution 4.0 International license. Further distribution of this work must maintain attribution to the author(s) and the published article's title, journal citation, and DOI. Funded by SCOAP. the electroweak scale, making them unreachable to current colliders. In this case, one is allowed to use effective field theory (EFT) techniques, integrate out the heavy states, and describe their impact at low energies by means of a collection of effective operators with canonical dimension larger than four. Following this procedure, in addition to the well-known dimension-five Weinberg operator that induces Majorana neutrinos, one usually obtains other nonrenormalizable operators with potentially observable effects in low-energy experiments.

In this work, we use bounds on the so-called neutrino nonstandard interactions (NSI) [2-6] derived at low-energy experiments to set constraints valid at high energies. In order to do that, we make use of the Standard Model effective field theory (SMEFT) $[7,8]$ and the low-energy effective field theory (LEFT) [9], two well-known EFTs valid at energies above or below the electroweak scale, respectively. The link between neutrino NSI and wellestablished EFTs, such as the LEFT and the SMEFT, allows one to study the phenomenology of a wide class of new physics (NP) scenarios in a model-independent way and easily confront results coming from a large diversity of experiments. In fact, this approach has been adopted in many recent works, deriving bounds from low-energy scattering [10] or reactor [11] experiments, or studying the interplay with searches for lepton flavor violating processes [12]. Lepton number violating NSI have been considered in the context of the SMEFT in [13], whereas a remarkable effort to provide a consistent EFT description of NSI has been made in [11,14]. The generalization of neutrino NSI including scalar or tensor couplings, the 
so-called neutrino generalized interactions (NGI) $[15,16]$, have also been discussed using an EFT language in [17].

Embedding NSI (or NGI) into EFTs as well established as the SMEFT and the LEFT provides a robust theoretical background and creates a direct link to other phenomenological directions. Among other advantages of this approach, one can easily compare the bounds obtained from neutrino NSI to bounds derived by other experimental probes. Here we will be interested in low-energy precision measurements and charged lepton flavor violation. We will systematically study a substantial region of the SMEFT parameter space with the help of DsixTools [18,19], a Mathematica package for the renormalization group equations (RGEs) running and matching in the SMEFT and the LEFT. This tool allows us to include full one-loop running effects (in the SMEFT and in the LEFT) in our numerical analysis. As a result of this, we will obtain robust numerical results and assess the relevance of RGE running for the NP scenarios considered in our study.

The rest of the paper is structured as follows. In Sec. II, we review the formalism and current experimental bounds on neutrino NSI. Section III introduces the SMEFT and the LEFT, the two EFTs considered in our analysis, while Sec. IV shows how these theories can be used in connection to neutrino NSI. Finally, we present our results in Sec. V and conclude in Sec. VI. Additional definitions are given in the Appendix.

\section{NEUTRINO NONSTANDARD INTERACTIONS}

As commented in the introduction, new neutrino vector interactions beyond the Standard Model can arise from neutrino mass models and other BSM theories. In the low-energy regime, neutrino NSI with matter fields can be formulated in terms of an effective four-fermion Lagrangian as follows:

$$
\begin{aligned}
\mathcal{L}_{\mathrm{CC}}^{\mathrm{NSI}}= & -\frac{G_{F}}{\sqrt{2}}\left(\epsilon_{p r}^{f f^{\prime} L}\left[\bar{\nu}_{p} \gamma^{\mu}\left(1-\gamma_{5}\right) e_{r}\right]\left[\bar{f}_{\mu}\left(1-\gamma_{5}\right) f^{\prime}\right]\right. \\
& \left.+\epsilon_{p r}^{f f^{\prime} R}\left[\bar{\nu}_{p} \gamma^{\mu}\left(1-\gamma_{5}\right) e_{r}\right]\left[\bar{f}_{\mu}\left(1+\gamma_{5}\right) f^{\prime}\right]\right)
\end{aligned}
$$

and

$$
\begin{aligned}
\mathcal{L}_{\mathrm{NC}}^{\mathrm{NSI}}= & -\frac{G_{F}}{\sqrt{2}}\left(\epsilon_{p r}^{f L}\left[\bar{\nu}_{p} \gamma^{\mu}\left(1-\gamma_{5}\right) \nu_{r}\right]\left[\bar{f}_{\mu}\left(1-\gamma_{5}\right) f\right]\right. \\
& \left.+\epsilon_{p r}^{f R}\left[\bar{\nu}_{p} \gamma^{\mu}\left(1-\gamma_{5}\right) \nu_{p}\right]\left[\bar{f}_{\mu}\left(1+\gamma_{5}\right) f\right]\right),
\end{aligned}
$$

where $\epsilon_{p r}^{f f^{\prime} L, R}$ and $\epsilon_{p r}^{f L, R}$ are dimensionless coefficients that quantify the strength of the NSI between neutrinos of flavor $p$ and $r$ and the matter field $f, f^{\prime}=u, d$ with $f \neq f^{\prime}$ for the case of charged current (CC)-NSI and $f=e, u, d$ for neutral current (NC)-NSI.

Neutrino NSI can affect experiments at the neutrino production via CC-NSI, changing the flavor distribution of the initial neutrino flux, and detection via both $\mathrm{CC}$ and $\mathrm{NC}$
NSI, depending on the detection technique of the experiment. Besides, neutrino NC-NSI can affect their propagation through matter as well, modifying the effective matter potential felt by neutrinos. In this work, we will concentrate on NC-NSI. ${ }^{1}$

The potential signal of NSI on neutrino experiments has been analyzed in detail in the literature recently [6,23-25]. The impact of this signal on the extraction of neutrino oscillation parameters from experimental data has also been extensively discussed; see, for instance Refs. [26-30]. However, since no signal of NSI has been experimentally reported yet, at the moment we only have upper bounds on their magnitude. These limits come from a variety of neutrino experiments, from oscillation experiments using solar, atmospheric, reactor or accelerator neutrino sources, to laboratory experiments measuring neutrino-electron and (coherent) neutrino-nucleus scattering. The size of the constraints on the NSI couplings depends on the neutrino flavors implied in the process, the most stringent one corresponding to the $\epsilon_{\mu \tau}^{d}$ coupling, bounded to be below $1 \%$ (at $90 \%$ C.L.) by the neutrino telescope IceCube [31].

From the point of view of particle physics models, NSI are mainly thought to come from interactions of an ultraviolet (UV) complete theory mediated by a heavy particle $X$ of mass $m_{X} \gg m_{\mathrm{EW}}$. Other alternative approaches to generate these new interactions have also been proposed. In particular, a possible explanation is to take the mass of the mediator particle much below the electroweak scale, $m_{X} \ll m_{\mathrm{EW}}$ [32-34]. This choice can avoid the strong bounds coming from charged lepton processes, linked to NSI due to gauge invariance [35]. This would allow the prediction of larger sizes of the NSI couplings, accessible to current or near future experiments. In any case, here we will focus on the first possibility, where the EFT approach applies.

For completeness, we would like to comment on the possibility of neutrino scalar and tensor four-fermion interactions, considered lately in the literature [15-17,36]. Note, however, that these NGI involve right-handed neutrinos and, therefore, they are not relevant to our study since, as we will discuss in the following section, our EFT analysis does not involve new particles.

\section{EFTs}

The SM of particle physics successfully describes a wide range of phenomena. However, it still leaves some questions unanswered, including the identity of the particle(s) accounting for dark matter or how neutrino masses are generated. For this reason, it is common to think of the SM as an effective theory valid up to a certain high-energy scale

\footnotetext{
${ }^{1}$ The effect of CC-NSI on reactor and long-baseline neutrino experiments has been discussed, for instance, in Refs. [20-22]. For a detailed analysis of CC-NSI in the context of EFTs, see [14].
} 
$\Lambda_{\mathrm{UV}}$, at which some unknown NP degrees of freedom would lie. The gauge group of a field theory valid above the electroweak scale should contain the SM gauge group and full particle content and, at energies below $\Lambda_{\mathrm{UV}}$, reduce to the SM. Generally, in most theories beyond the SM, this reduction occurs via decoupling of heavy particles with masses of order $\Lambda_{U V}$ or larger. This leads to the appearance of higher dimensional operators in the SM Lagrangian suppressed by powers of $\Lambda_{\mathrm{UV}}$. The EFT built with all the operators that respect the SM gauge group with dimension $d \geq 5$ is the SMEFT. The SMEFT Lagrangian is given by

$$
\mathcal{L}_{\mathrm{SMEFT}}=\mathcal{L}_{\mathrm{SM}}+\sum_{d \geq 5} \mathcal{L}_{\mathrm{SMEFT}}^{(d)},
$$

with

$$
\mathcal{L}_{\mathrm{SMEFT}}^{(d)}=\sum_{i=1}^{n_{d}} \frac{C_{i}^{(d)}}{\Lambda_{\mathrm{UV}}^{d-4}} Q_{i}^{(d)},
$$

where $\mathcal{L}_{\mathrm{SM}}$ is the SM Lagrangian, $Q_{i}^{(d)}$ are operators of dimension $d$, and $C_{i}^{(d)}$ the corresponding Wilson coefficients (WCs). We note that the $C_{i}^{(d)}$ WCs have been defined as dimensionless quantities by making explicit the suppression by the high-energy scale $\Lambda_{\mathrm{UV}}$. The full set of SMEFT operators up to dimension six was given in [8], defining the so-called Warsaw basis. Finally, the complete one-loop anomalous dimension matrix for the dimensionsix operators in this basis was obtained in [37-40]. This describes the energy evolution of the Wilson coefficients as

$$
\mu \frac{d C_{i}}{d \mu}=\frac{1}{16 \pi^{2}} \sum_{j} \gamma_{i j}^{S} C_{j},
$$

where $\mu$ is the renormalization scale and $\gamma^{S}$ the anomalous dimension matrix for the operators of the SMEFT. Among all the operators of the SMEFT, we list the most relevant operators for the study of neutrino NSI ( $p, r, s, t$ are flavor indices) which are as follows:

$$
\begin{aligned}
& \text { - } Q_{p r s t}=\left[\bar{\ell}_{p} \gamma^{\mu} \ell_{r}\right]\left[\bar{\ell}_{s} \gamma_{\mu} \ell_{t}\right] \cdot Q_{\text {prst }}=\left[\bar{\ell}_{p} \gamma^{\mu} \ell_{r}\right]\left[\bar{u}_{s} \gamma_{\mu} u_{t}\right]
\end{aligned}
$$

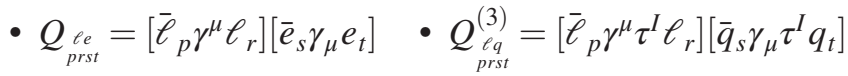

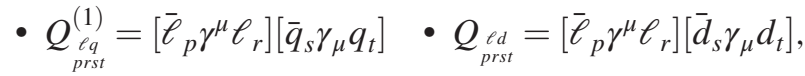

where $\ell$ and $q$ are the SM lepton and quark doublets, and $e$, $u$, and $d$ the singlets.

Neutrino experiments mainly deal with energies way below the electroweak scale. Therefore, a new effective theory is needed to describe low-energy processes. This EFT can be derived from the SM by integrating out the massive electroweak gauge bosons $\left(W^{ \pm}, Z\right)$, the Higgs boson, and the chiral top quark fermion fields $\left(t_{L}\right.$ and $\left.t_{R}\right)$.
The gauge group of this LEFT is $\mathrm{SU}(3)_{\mathrm{C}} \times \mathrm{U}(1)_{\mathrm{Q}}$, i.e., the symmetry of QCD and QED. The LEFT Lagrangian reads

$$
\mathcal{L}_{\mathrm{LEFT}}=\mathcal{L}_{\mathrm{QCD}+\mathrm{QED}}+\mathcal{L}_{\psi}^{(3)}+\sum_{d \geq 5} \mathcal{L}_{\mathrm{LEFT}}^{(d)}
$$

The first term contains the QCD gauge interaction for two families of up quarks and three of down quarks, the QED gauge interaction for these quarks and the three charged lepton families, and their Dirac mass terms,

$$
\begin{aligned}
\mathcal{L}_{\mathrm{QCD}+\mathrm{QED}}= & -\frac{1}{4} G_{\mu \nu}^{\alpha} G^{\alpha, \mu \nu}-\frac{1}{4} F_{\mu \nu} F^{\mu \nu}+\theta_{s} \frac{g_{s}^{2}}{32 \pi^{2}} G_{\mu \nu}^{a} \tilde{G}^{a, \mu \nu} \\
& +\theta_{\mathrm{QED}} \frac{e^{2}}{32 \pi^{2}} F_{\mu \nu} \tilde{F}^{\mu \nu} \\
& +\sum_{\psi=e, \nu_{L}, u, d} i \bar{\psi} \gamma^{\mu} D_{\mu} \psi-\sum_{\psi=e, u, d} \bar{\psi}_{R r}\left[M_{\psi}\right]_{r s} \psi_{L s} \\
& - \text { H.c. },
\end{aligned}
$$

where $r, s$ are flavor indices and $D_{\mu} \psi=\left(\partial_{\mu}-i Q A_{\mu}-\right.$ $\left.i g_{s} T_{s}^{a} G_{\mu}^{a}\right)$. We note that the three left-handed neutrinos are gauge singlets with no Dirac mass term. The second term in Eq. (6) consists of $\Delta L= \pm 2$ Majorana mass terms for the left-handed neutrinos,

$$
\mathcal{L}_{\psi}^{(3)}=-\frac{1}{2}\left[M_{\nu}\right]_{r s}\left(\nu_{L r}^{T} C \nu_{L s}\right)+\text { H.c. }
$$

Here $M_{\nu}^{T}=M_{\nu}$ is the symmetric Majorana mass matrix. In the case of three flavors of neutrinos, there will be six different $\Delta L=2$ operators and six conjugate $\Delta L=-2$ operators. Finally, the last piece contains operators of dimension five or higher,

$$
\mathcal{L}_{\mathrm{LEFT}}^{(d)}=\sum_{i=1}^{n_{d}} \frac{L_{i}^{(d)}}{v^{d-4}} \mathcal{O}_{i}^{(d)},
$$

where $\mathcal{O}_{i}^{(d)}$ are dimension $d$ operators and $L_{i}^{(d)}$ their WCs coefficients. Again, the $L_{i}^{(d)}$ WCs have been defined as dimensionless quantities by introducing an explicit suppression by $1 / v^{d-4}$, where $v$ is the Higgs vacuum expectation value that sets the electroweak scale. The full set of LEFT operators up to dimension six and their tree-level matching relations with the SMEFT operators can be found in [9]. ${ }^{2}$ We will stick to this basis of operators, referred to as the San Diego basis. The complete one-loop anomalous dimension matrix for this basis of LEFT operators, $\gamma^{L}$, was derived in [42], such that

\footnotetext{
${ }^{2}$ We note that the one-loop SMEFT-LEFT matching relations were recently derived in [41], although we will not use them in our analysis.
} 


$$
\mu \frac{d L_{i}}{d \mu}=\frac{1}{16 \pi^{2}} \sum_{j} \gamma_{i j}^{L} L_{j}
$$

describes the evolution of the $L_{i}$ WCs with the renormalization scale $\mu$. We now list some LEFT operators of relevance for the study of neutrino NC NSI which are as follows:

$$
\begin{aligned}
& \text { - } \mathcal{O}_{\substack{\nu, L L \\
\text { prst }}}^{V, L L}=\left[\bar{\nu}_{L, p} \gamma^{\mu} \nu_{L, r}\right]\left[\bar{u}_{L, s} \gamma_{\mu} u_{L, t}\right] \\
& \text { - } \mathcal{O}_{\substack{\nu, L R \\
p r s t}}^{V, L R}=\left[\bar{\nu}_{L, p} \gamma^{\mu} \nu_{L, r}\right]\left[\bar{u}_{R, s} \gamma_{\mu} u_{R, t}\right] \\
& \text { - } \mathcal{O}_{\substack{p d \\
p r s t}}^{V, L L}=\left[\bar{\nu}_{L, p} \gamma^{\mu} \nu_{L, r}\right]\left[\bar{d}_{L, s} \gamma_{\mu} d_{L, t}\right] \\
& \text { - } \mathcal{O}_{\substack{\nu d \\
p r s t}}^{V, L R}=\left[\bar{\nu}_{L, p} \gamma^{\mu} \nu_{L, r}\right]\left[\bar{d}_{R, s} \gamma_{\mu} d_{R, t}\right] \\
& \text { - } \mathcal{O}_{\substack{\nu, L L \\
\text { prst }}}^{V, L L}=\left[\bar{\nu}_{L, p} \gamma^{\mu} \nu_{L, r}\right]\left[\bar{e}_{L, s} \gamma_{\mu} e_{L, t}\right] \\
& \text { - } \mathcal{O}_{\substack{\nu, L R \\
p r s t}}^{V, L R}=\left[\bar{\nu}_{L, p} \gamma^{\mu} \nu_{L, r}\right]\left[\bar{e}_{R, s} \gamma_{\mu} e_{R, t}\right] \text {, }
\end{aligned}
$$

where $\nu_{L}, e_{L / R}$, and $u_{L / R}$ and $d_{L / R}$ are the chiral left-/righthanded neutrino, charged lepton, and up-quark and downquark fields.

The electroweak scale sets the limit between the SMEFT and the LEFT and determines the energy scale at which these two theories must be matched by integrating out the $W$ and $Z$ gauge bosons, the Higgs boson, and the top quark. When doing so, one must take into account the breaking of the electroweak symmetry, therefore matching the SMEFT in the broken phase with the LEFT. ${ }^{3}$ An obvious feature arising from this matching will be the breaking of the $\mathrm{SU}(2)_{\mathrm{L}}$ doublets, originating several LEFT operators from a single SMEFT operator. For example, from the SMEFT operator $Q_{\ell \ell}$, the LEFT operators $\mathcal{O}_{\nu e}^{V, L L}, \mathcal{O}_{e e}^{V, L L}$, and $\mathcal{O}_{\nu \nu}^{V, L L}$ will emerge. Furthermore, the LEFT operators will receive several contributions. In addition to those originated from the dimension-six SMEFT operators, pure SM contributions exist as well. For instance, the matching relation for the $\mathcal{O}_{\nu \nu}^{V, L L}$ LEFT operator is

$\frac{L_{\substack{\nu, L L \\ p r s t}}^{V, L L}}{v^{2}}=\frac{C_{\ell \ell} p r s t}{\Lambda_{\mathrm{UV}}^{2}}-\frac{\bar{g}_{Z}^{2}}{4 M_{Z}^{2}}\left[Z_{\nu}\right]_{p r}\left[Z_{\nu}\right]_{s t}-\frac{\bar{g}_{Z}^{2}}{4 M_{Z}^{2}}\left[Z_{\nu}\right]_{p t}\left[Z_{\nu}\right]_{s r}$,

where $p, r, s, t$ are flavor indices. The first term constitutes the contribution of the SMEFT operator $Q_{\ell \ell}$, whereas the last two terms correspond to two contributions to the $\mathcal{O}_{\nu \nu}^{V, L L}$ LEFT operator obtained by $Z$ boson exchange. $\left[Z_{\nu}\right]$ is the $Z$ coupling to a pair of neutrinos which, in addition to the pure SM coupling, contains contributions from the SMEFT operators $Q_{H \ell}^{(1)}$ and $Q_{H \ell}^{(3)}$. Finally, $\bar{g}_{Z}$ is an effective coupling containing the contribution of

\footnotetext{
${ }^{3}$ The Appendix compiles the most relevant analytical expressions for the SM parameters including their modifications in the presence of contributions from dimension-six SMEFT operators.
}

dimension-six Higgs-gauge-boson operators $X^{2} H^{2}$. Equation (11) assumes the SMEFT WCs to be given in the fermion up basis, defined by diagonal up-quark and charged lepton Yukawa matrices, since this basis allows one to identify the top quark, one of the fields integrated out at this stage. We will adopt this implicit assumption in all the matching relations given in this paper and omit the unitary matrices that transform to the up basis in order to simplify the resulting expressions. The full set of SMEFTLEFT tree-level matching relations can be found in [9]. ${ }^{4}$

\section{NEUTRINO NSI IN THE LEFT AND THE SMEFT}

After discussing neutrino NSI and two EFTs of interest, the SMEFT and the LEFT, we proceed to establish a link between them. This will allow us to study neutrino NSI in the language of the SMEFT and the LEFT and, more importantly, to make use of the theoretical machinery developed for these two theories. In fact, as shown in Sec. II, neutrino NSI are encoded by a set of coefficients of low-energy effective operators. Therefore, the link to the LEFT is quite straightforward. One can find a one-to-one relation between the NSI effective operators and the LEFT operators which, in turn, can be matched to the SMEFT operators valid at high energies. In Table I, we list all the NC NSI coefficients and their matching with the LEFT and SMEFT WCs. This table makes use of the definitions in Appendix, which are taken from [9]. As explained in Sec. III, the SMEFT WCs are assumed to be given in the fermion up basis. The relevant unitary matrices involved in the transformation to this basis are not explicitly indicated to simplify the notation. ${ }^{5}$ Furthermore, we note that the SMEFT-LEFT matching relations in Table I include pure SM contributions. These must be removed in the final matching to the NSI coefficients to properly identify the nonstandard pieces.

Armed with these matching relations and the RGEs of both EFTs, we can bring the bounds coming from low (high)-energy experiments to high (low) energies and translate them to the most convenient effective theory in each case. In particular, the main goal of our work is to use NC neutrino NSI to derive limits on the SMEFT WCs at high energies. One could naively think that the best method to do this is to start at low energies, match the NSI coefficients to the LEFT WCs, run up to the EW scale,

\footnotetext{
${ }^{4}$ At energies below $\mu \sim 5 \mathrm{GeV}$, one should adopt other EFTs, better suited to take into account the nonperturbative nature of the strong interactions in this energy regime (see, for instance, $[11,43])$. In order to simplify our study and be able to ignore this issue, we will never run below $5 \mathrm{GeV}$ and neglect this possibility.

${ }^{5}$ Alternatively, [17] gives analogous matching relations in the down basis, in which the down-quark Yukawa matrix is diagonal. This reference also includes explicitly the quark mixing matrices appearing in the transformation to the down basis.
} 
TABLE I. Tree-level matching of the NC NSI coefficients (with flavor indices $p$ and $r$ ) to the LEFT and SMEFT Wilson coefficients. The SMEFT-LEFT matching relations were derived in [9]. The SMEFT WCs are assumed to be given in the up fermion basis; see Sec. III for details. The pure SM contributions are removed in the final matching to the NSI coefficients. We refer to the Appendix for notation and conventions.

\begin{tabular}{|c|c|c|}
\hline NSI & LEFT & SMEFT \\
\hline$-2 \sqrt{2} G_{F} \epsilon_{p r}^{u L}$ & $\frac{1}{v^{2}} L_{\substack{v u \\
p r 11}}^{V, L L}$ & $\frac{1}{\Lambda_{\mathrm{UV}}^{2}}\left(C_{\ell q}^{(1)}+C_{p q}^{(3)}\right)-\frac{\bar{g}_{Z}^{2}}{M_{Z}^{2}}\left[Z_{\nu}\right]_{p r}\left[Z_{u_{L}}\right]_{11}$ \\
\hline$-2 \sqrt{2} G_{F} \epsilon_{p r}^{d L}$ & $\frac{1}{v^{2}} L_{\substack{v d \\
p r 11}}^{V, L L}$ & $\frac{1}{\Lambda_{\mathrm{UV}}^{2}}\left(C_{\ell q}^{(1)}-C_{p q 11}^{(3)}\right)-\frac{\bar{g}_{Z}^{2}}{M_{Z}^{2}}\left[Z_{\nu}\right]_{p r}\left[Z_{d_{L}}\right]_{11}$ \\
\hline$-2 \sqrt{2} G_{F} \epsilon_{p r}^{e L}$ & $\frac{1}{v^{2}} L_{\substack{v e \\
p r 11}}^{V, L L}$ & $\begin{array}{c}\frac{1}{\Lambda_{\mathrm{UV}}^{2}}\left(C_{p r 11}+C_{\substack{\ell \ell p \\
p r}}\right)-\frac{\bar{g}_{2}^{2}}{2 M_{W}^{2}}\left[W_{\ell}\right]_{p 1}\left[W_{\ell}\right]_{r 1}^{*}- \\
\frac{\bar{g}_{Z}^{2}}{M_{Z}^{2}}\left[Z_{\nu}\right]_{p r}\left[Z_{e_{L}}\right]_{11}\end{array}$ \\
\hline$-2 \sqrt{2} G_{F} \epsilon_{p r}^{u R}$ & $\frac{1}{v^{2}} L_{p r 11}^{V, L R}$ & $\frac{1}{\Lambda_{\mathrm{UV}}^{2}} C_{p r 11}-\frac{\bar{g}_{Z}^{2}}{M_{Z}^{2}}\left[Z_{\nu}\right]_{p r}\left[Z_{u_{R}}\right]_{11}$ \\
\hline$-2 \sqrt{2} G_{F} \epsilon_{p r}^{d R}$ & $\frac{1}{v^{2}} L_{\substack{v d \\
p r 11}}^{V, L R}$ & $\frac{1}{\Lambda_{\mathrm{UV}}^{2}} C_{p r d 1}{ }_{p d}-\frac{\bar{g}_{Z}^{2}}{M_{Z}^{2}}\left[Z_{\nu}\right]_{p r}\left[Z_{d_{R}}\right]_{11}$ \\
\hline$-2 \sqrt{2} G_{F} \epsilon_{p r}^{e R}$ & $\frac{1}{v^{2}} L_{\substack{v e \\
p r 11}}^{V, L R}$ & $\frac{1}{\Lambda_{\mathrm{UV}}^{2}} C_{p e r 11}-\frac{\bar{g}_{Z}^{2}}{M_{Z}^{2}}\left[Z_{\nu}\right]_{p r}\left[Z_{e_{R}}\right]_{11}$ \\
\hline
\end{tabular}

match the LEFT to the SMEFT, and finally run up to the high-energy scale $\Lambda_{\mathrm{UV}}$, where the resulting limit is obtained. However, this method is inconsistent because it does not take into account that the LEFT is more general than the SMEFT. For instance, a low-energy scenario with a single NSI coefficient or, equivalently, with a single LEFT WC, might not be consistent with a high-energy SMEFT origin, since $\mathrm{SU}(2)_{\mathrm{L}}$ gauge invariance imposes relations between LEFT WCs after SMEFT-LEFT matching. For this reason, running up from low energies is not (in general) a consistent approach to achieve our goal. Instead, one must do the opposite: to consider a SMEFT parameter point at high energies, run down to the EW scale, match to the LEFT, continue running down to low energies, match to the NSI coefficients, and compare the resulting values to the experimental bounds. This process can be repeated for different input SMEFT scenarios and high-energy scales, thus determining the region of the SMEFT parameter space that is compatible with the low-energy NSI bounds.
We will now illustrate our procedure with an explicit example.

\section{A. An example}

To make more explicit the method used in our analysis, we will now follow step by step the full path that takes from the high-energy SMEFT to the NSI coefficients that play a role in neutrino experiments. Let us study a process of interest such as a hypothetical new interaction between electron neutrinos and left-handed electrons.

First, we start at the high-energy scale $\Lambda_{\mathrm{UV}}$, where the $\mathrm{SU}(2)_{\mathrm{L}}$ symmetry is unbroken, and consider an interaction involving the first generation lepton doublet, $\ell_{e}$, induced by the exchange of an unknown heavy vector mediator, $X$, with $m_{X} \sim \Lambda_{\mathrm{Uv}}$. At energies below $m_{X}$, the tree-level exchange of the heavy $X$ vector can be effectively described by a four-fermion interaction, with strength $\tilde{g}_{\ell \ell} \cdot \tilde{g}_{\ell \ell}$, where $\tilde{g}_{\ell \ell}$ is the coupling of $X$ to a pair of lepton doublets, and suppressed by $\frac{1}{\Lambda_{\mathrm{UV}}^{2}}$. In Fig. 1, we can see a diagrammatic representation of this. There, we see that integrating out $X$ leads to the generation of the $4=$ four-fermion SMEFT operator $Q_{\ell \ell}=\left[\bar{\ell}_{p} \gamma^{\mu} \ell_{r}\right]\left[\bar{\ell}_{s} \gamma_{\mu} \ell_{t}\right]$, with Wilson coefficient $C_{\ell \ell}=\tilde{g}_{\ell \ell} \cdot \tilde{g}_{\ell \ell}$ and suppressed by $1 / \Lambda_{\mathrm{UV}}^{2}$. We can now solve the SMEFT RGEs to obtain the SMEFT Lagrangian at the electroweak scale, where the SM symmetry breaks and the SMEFT must be matched to the LEFT. With only one nonvanishing input SMEFT WC, the main contributions to the RGEs come from the terms proportional to it. For the case under discussion, the main term is

$$
\dot{C}_{\substack{\ell 11 \\ 111}} \sim 2\left[Y_{e}^{\dagger} Y_{e}\right]_{11} C_{\ell \ell} .111 .
$$

The next step is to match the SMEFT with the LEFT. As already discussed, from a single SMEFT operator, one gets several LEFT operators. In this case, since we are interested in electron neutrino-electron interactions, we focus on $\mathcal{O}_{\nu e}^{V, L L}$. We are then studying a four-fermion diagram with two electron neutrinos and two left-handed electrons. An important feature to take into account is that this diagram is
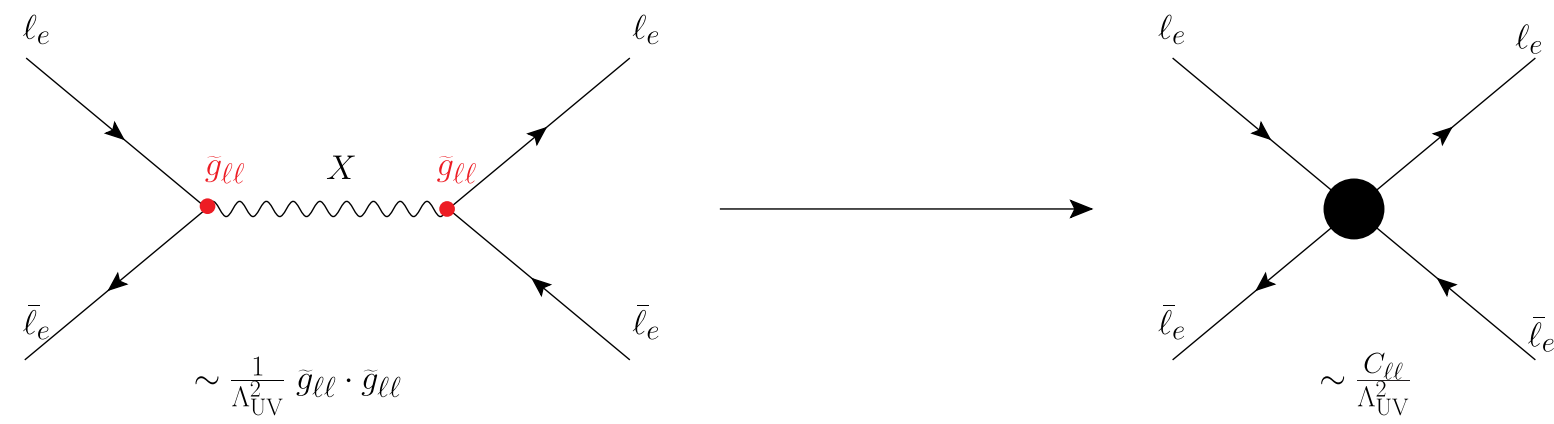

FIG. 1. Feynman diagram of the process $\ell_{e} \bar{\ell}_{e} \rightarrow \ell_{e} \bar{\ell}_{e}$ via the exchange of an unknown heavy mediator that gives rise to a dimensionsix effective operator in the SMEFT. 
not only generated by the previously discussed dimensionsix SMEFT operator, but also from pure SM diagrams with $W$ and $Z$ bosons exchange, as we can see in Fig. 2. The tree-level matching relation between this LEFT operator and the SMEFT is given by

$\frac{L_{\substack{\nu, L \\ 1111}}^{V, L L}}{v^{2}}=2 \frac{C_{\ell \ell}}{\Lambda_{\mathrm{UV}}^{211}}-\frac{\bar{g}_{2}^{2}}{2 M_{W}^{2}}\left[W_{\ell}\right]_{11}\left[W_{\ell}\right]_{11}^{*}-\frac{\bar{g}_{Z}^{2}}{M_{Z}^{2}}\left[Z_{\nu}\right]_{11}\left[Z_{e_{L}}\right]_{11}$,

where $\left[W_{\ell}\right]$ and $\left[Z_{\nu / e_{L}}\right]$ are the $W$ and $Z$ couplings to neutrinos/electrons and $\bar{g}_{2}^{2}$ and $\bar{g}_{Z}^{2}$ quantify the strength of the $W$ and $Z$ interactions. Explicit expressions for these couplings, including the corrections due to dimension-six operators, are given in the Appendix.

After the SMEFT-LEFT matching, we solve the RGEs down to the low-energy scale $\Lambda_{\mathrm{IR}}$, where the NSI bounds are set. For the operator we are interested in, the main RGE term is

$$
\dot{L}_{\substack{L e \\ 1111}}^{V, L L} \sim \frac{4}{3} e^{2} L_{\substack{\nu l \\ 11 r}}^{V, L L}
$$

where we sum over the flavor index $r$. Finally, we perform the matching between the LEFT and NSI operators. In this
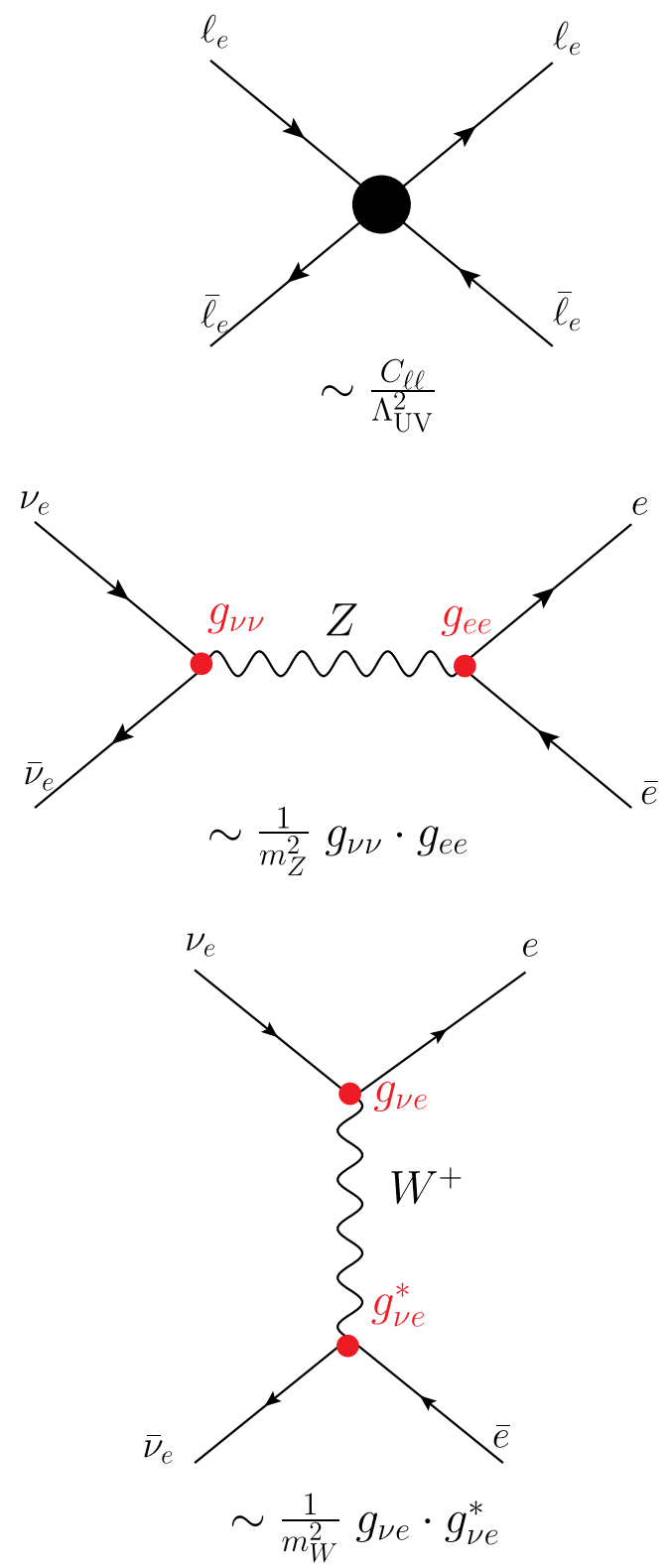
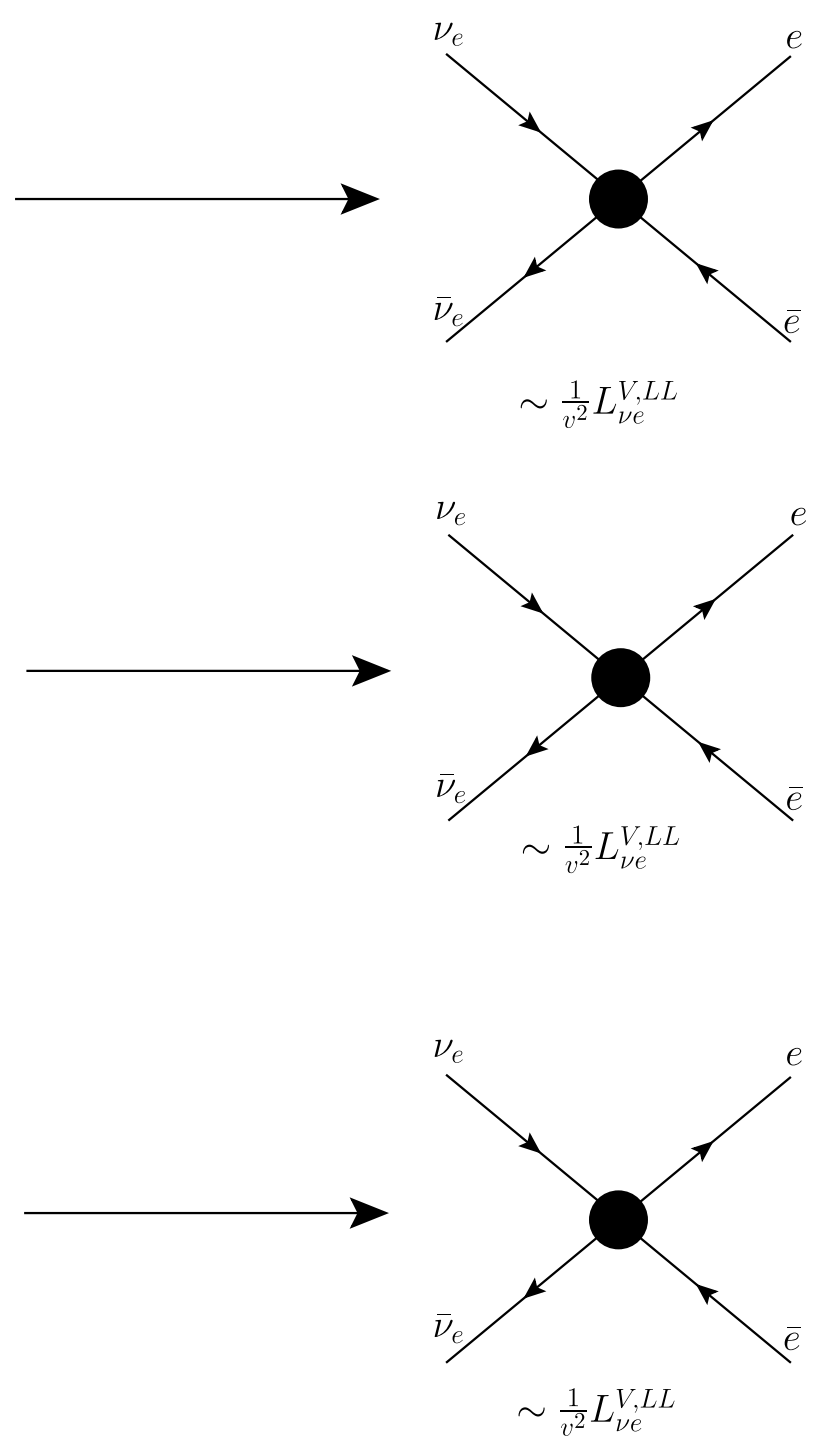

FIG. 2. Feynman diagrams that contribute to the process $\ell_{e} \bar{\ell}_{e} \rightarrow \ell_{e} \bar{\ell}_{e}$ in the SMEFT at tree-level, both from dimension-six operators and from gauge boson exchange, giving rise to a dimension-six effective operator in the LEFT. 
case, the relation between the coefficients is quite simple, as shown in Table I. For the example we are going through, the matching is given by

$$
\epsilon_{e e}^{e L}=-\frac{\left(L_{\nu, L}^{V, L L}\right)^{\mathrm{BSM}}}{2 \sqrt{211} G_{F} v^{2}},
$$

where $\left(L_{\substack{L e \\ \text { Le }}}^{V, L L}\right)^{\mathrm{BSM}}$ is the pure BSM contribution to the LEFT WC $L_{\substack{\nu L \\ 1111}}^{V, L L}$. After this plain matching, we get a value for the NSI coefficient we are interested in at a certain energy scale, ready to be compared with the experimental bounds.

\section{NUMERICAL ANALYSIS}

Having set our notation and described our strategy, we now proceed to show the results of our numerical analysis. In order to explore a substantial region of the huge SMEFT parameter space, we have considered a large number of SMEFT operators (with specific flavor indices) and applied the approach discussed in Sec. IV. This way, we have been able to map a region of interest in the SMEFT onto the NSI parameter space, where the experimental constraints previously derived in the literature can be directly applied.

A total of 112 initial nonzero SMEFT WCs have been selected. For each of them, and assuming only one at a time, we have considered 14 different values for the NP scale, $\Lambda_{\mathrm{UV}}$, in the $[0.5,14] \mathrm{TeV}$ range. This NP scale not only sets the starting point for the RGE running, but also the value of the SMEFT WC, taken to be precisely $\left|C_{i}\right|=1$ at $\mu=\Lambda_{\mathrm{UV}}$. Then, as a result of the strategy explained in Sec. IV, 48 NSI coefficients are obtained at the low-energy scale $\Lambda_{\mathrm{IR}}=5 \mathrm{GeV}$ for each scenario. This includes NC NSI with both chiralities. We finally compare these values with the current NSI experimental bounds and derive limits for the original SMEFT WCs at high energies.

Our numerical calculations have been obtained with the help of DsixTools [18,19]. This Mathematica package has several tools and functionalities for the RGE running and matching in the SMEFT and the LEFT and is perfectly suited for our phenomenological exploration. In particular, we used version 2.0 [19], which fully integrates the LEFT, and only added the matching between the LEFT and NSI operators. This approach allows one to explore the relation between neutrino NSI, the LEFT and the SMEFT in a systematic way. To the best of our knowledge, our work is the first to study such connection including full one-loop running effects. We use DsixTools to solve the LEFT RGEs numerically while the SMEFT RGEs are solved following a semianalytical approach based on an evolution matrix formalism [44]. We take advantage of one of the main DsixTools functionalities: user-friendly input and output, which can be given in the DsixTools native format as well as using the WCXf exchange format [45]. All input parameters will be assumed to be specified in the up basis.
We have explicitly checked that the charged lepton Yukawa matrix remains in very good approximation diagonal after RGE running at high and low energies. This allows for an easy identification of the neutrino flavor eigenstates, precisely defined by the basis in which the charged lepton Yukawas are diagonal. Furthermore, DsixTools transforms all SMEFT parameters to the up basis before applying the matching relations of Ref. [9].

In what concerns the experimental NSI limits used in our analysis, these come from various sources, including neutrino oscillation and scattering experiments. We have used the bounds compiled in [6], where an extensive review of the NSI formalism and experimental limits is done. More precisely, the most relevant couplings for our analysis come from the following:

(i) Analysis of neutrino-nucleon scattering data $[24,46]\left(\epsilon_{e \mu}^{q L}\right)$

(ii) Combined analysis of atmospheric and neutrinonucleon scattering data [46] $\left(\epsilon_{\mu \mu}^{d V}\right)$

(iii) Analysis of the atmospheric neutrino signal in IceCube DeepCore [31] $\left(\epsilon_{\mu \tau}^{q V}\right)$

(iv) Combined analysis of solar and KamLAND reactor data [47] $\left(\epsilon_{e e}^{e L}\right)$

(v) Combined analysis of reactor and accelerator data $[48,49]\left(\epsilon_{\mu \mu}^{e L}\right)$

(vi) Combination of oscillation and coherent neutrinonucleus scattering data [50] $\left(\epsilon_{e e}^{u V}\right)$

(vii) Analysis of atmospheric neutrino data $[6,51]\left(\epsilon_{\tau \tau}^{q V}\right)$

\section{A. Neutrino NSI from the SMEFT at high energies}

Before moving to the discussion of the limits on SMEFT WCs derived from neutrino NSI, it is illustrative to show some selected examples of the NSI coefficients generated by several SMEFT scenarios. We can visualize these results by plotting the values of the NSI coefficients obtained for a certain initial nonzero SMEFT WC at several scales along with the experimental bounds on the NSI coefficients. This way we can easily determine whether a specific SMEFT scenario is constrained or not due to neutrino NSI for a given $\Lambda_{\mathrm{UV}}$. A first example of this strategy is presented in Fig. 3. This figure displays the values of the left chiral NC NSI coefficients arising from the SMEFT WC $C_{\substack{l q \\ 121}}^{(1)}$. Three possible $\Lambda_{\mathrm{UV}}$ values are considered, $0.5,1$, and $2 \mathrm{TeV}$. As expected, lower NP scales imply larger NSI coefficients. The current experimental limits on the different NSI coefficients are indicated with red lines, implying the exclusion of any SMEFT parameter point leading to NSI coefficients at low energies that fall outside of them. For instance, in this example, we find that the bound on $\epsilon_{e \mu}^{u L}$ excludes $\Lambda_{\mathrm{UV}} \lesssim 1 \mathrm{TeV}$. Also, this figure seems to indicate that only two NSI coefficients are generated at $\Lambda_{\mathrm{IR}}$. Actually, since we are including full one-loop running effects in our calculation, many NSI coefficients are nonvanishing at low energies. For this particular example, all 


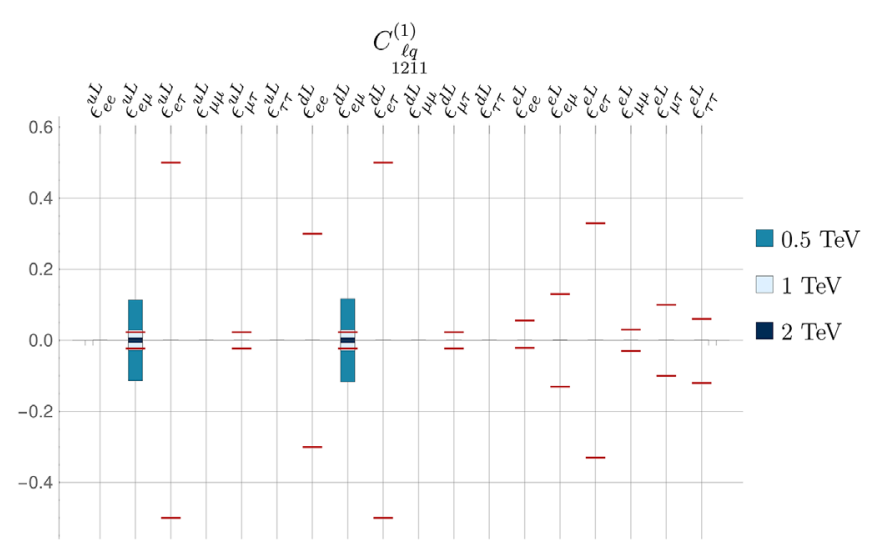

FIG. 3. Values for the various chiral left NSI coefficients obtained assuming the SMEFT WC $\left|C_{t q}^{(1)}\right|=1$ at $\mu=\Lambda_{\mathrm{UV}}$ for different values of $\Lambda_{\mathrm{UV}}$. The red lines correspond to the experimental bounds on the NSI coefficients compiled in [6].

$\mu-e$ flavor violating NSI coefficients are generated. However, most of them are too small to be visualized in Fig. 3 and only $\epsilon_{e \mu}^{u L}$ and $\epsilon_{e \mu}^{d L}$, the two $\mu-e$ flavor violating NSI coefficients with first generation left-handed quarks, have sizable values. In fact, the sizable values obtained for these two NSI coefficients could have been predicted just by using the tree-level matching relation

$$
\epsilon_{e \mu}^{u L} \simeq \epsilon_{e \mu}^{d L} \sim-\frac{C_{\ell q}^{(1)}}{1211}, \frac{\sqrt{21}}{2 G_{F} \Lambda_{\mathrm{UV}}^{2}},
$$

given in Table I. This approximate relation is reproduced in our numerical results. Moreover, other NSI coefficients would only be generated due to operator mixing effects. Since they have tiny values, we conclude that operator mixing effects are negligible in this scenario.

One can also find SMEFT scenarios leading to NSI coefficients compatible with the current limits even for NP scales as low as $\Lambda_{\mathrm{UV}}=0.5 \mathrm{TeV}$. ${ }^{6}$ This is the case when the input SMEFT WC involves only second or third generation quarks. Even though nonvanishing NSI coefficients with first generation quarks are obtained due to quark mixing effects in the RGEs, these are always tiny. Therefore, scenarios of this sort will not be considered in our subsequent analysis, since they cannot be effectively bounded by neutrino NSI. Similarly, there are scenarios leading to sizable NSI, but not large enough to be constrained. This is, for instance, illustrated in Fig. 4, which shows the left chiral NC NSI coefficients arising from the input SMEFT WC $C_{\ell \ell}$. The only non-negligible NSI coefficient in this case is $\epsilon_{\tau \tau}^{e L}$. The joint analysis of solar neutrino experiments (mostly Super-Kamiokande) and

\footnotetext{
${ }^{6} \Lambda_{\mathrm{UV}}=0.5 \mathrm{TeV}$ is the lowest NP scale considered in our analysis. Below that value the SMEFT approach is no longer justified.
}

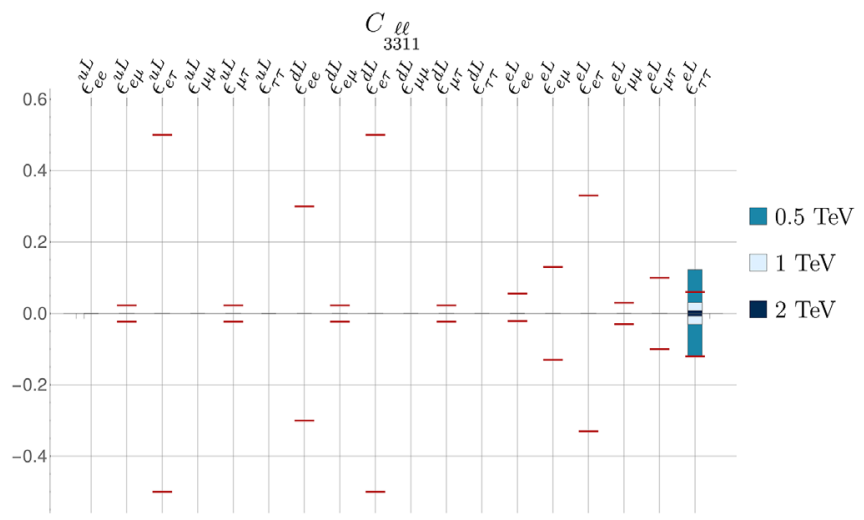

FIG. 4. Values for the various chiral left NSI coefficients obtained assuming the SMEFT WC $\left|C_{\ell \ell}\right|=1$ at $\mu=\Lambda_{\mathrm{UV}}$ for different values of $\Lambda_{\mathrm{UV}}$. The red lines correspond to the experimental bounds on the NSI coefficients compiled in [6].

KamLAND requires $-0.12<\epsilon_{\tau \tau}^{e L}<0.06$ at $90 \%$ C.L. Due to the asymmetry in these experimental limits, scenarios with $C_{3311}<0$ require $\Lambda_{\mathrm{UV}}$ to be above $\sim 0.7 \mathrm{TeV}$, while the NP scale can be as low as $0.5 \mathrm{TeV}$ when $C_{3311}>0$. Therefore, in this case, no relevant bound on $\Lambda_{\mathrm{UV}}$ can be obtained. Finally, there are also scenarios for which the current experimental limits on the generated NSI coefficients turn out to be too weak. An example of this situation is shown in Fig. 5, where we plot the NSI coefficients obtained from the initial SMEFT WC $C_{\substack{1 q \\ 1311}}^{(1)}$. The largest NSI coefficients in this case are $\epsilon_{e \tau}^{u L}$ and $\epsilon_{e \tau}^{d L}$, and these are only very weakly constrained.

In general, we have found that SMEFT scenarios with $\Lambda_{\mathrm{UV}}>3 \mathrm{TeV}$ lead to tiny NSI coefficients at low energies, always in agreement with the current experimental bounds. For this reason, the rest of the analysis will concentrate on NP scales between 0.5 and $3 \mathrm{TeV}$. Moreover, the previous

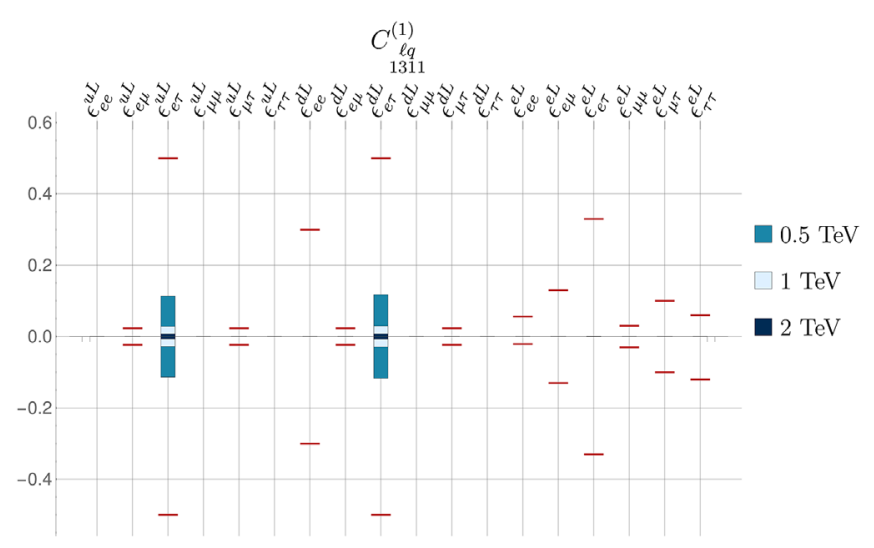

FIG. 5. Values for the various chiral left NSI coefficients obtained assuming the SMEFT WC $\left|C_{\ell q}^{(1)}\right|=1$ at $\mu=\Lambda_{\mathrm{UV}}$ for different values of $\Lambda_{\mathrm{UV}}$. The red lines correspond to the experimental bounds on the NSI coefficients compiled in [6]. 
exploration allowed us to identify the SMEFT scenarios capable to generate sizable NSI coefficients, potentially resulting in relevant limits on the SMEFT WCs. Obtaining these limits is our next goal.

\section{B. Limits on SMEFT Wilson coefficients from neutrino NSI}

After our previous exploration, we have identified 18 SMEFT scenarios that give sizeable NSI coefficients for NP scales $\gtrsim 1 \mathrm{TeV}$ and performed again the procedure described in Sec. IV for each of them. After evaluating the resulting NSI coefficients at low energies assuming $\left|C_{i}\right|=$ 1 at $\mu=\Lambda_{\mathrm{UV}}$ for different $\Lambda_{\mathrm{UV}}$ values, one can easily interpolate to determine the value of the NP scale that corresponds to the experimental bound of the NSI coefficient, thus setting in this way a lower bound on the combination $\Lambda_{\mathrm{UV}} / \sqrt{\left|C_{i}\right|}$ for that particular SMEFT WC.

The bounds obtained with this method range between $\sim 700 \mathrm{GeV}$ and $\sim 2.1 \mathrm{TeV}$. We observe that the flavor violating operators involving the second and third lepton generations get the strongest bounds. This is because the most restrictive neutrino NSI experimental bound, compiled in Ref. [6], is the NC NSI involving quarks and the muon and tau neutrinos. This bound, derived from IceCube DeepCore data [31], sets the lower limit of $\Lambda_{\mathrm{UV}} / \sqrt{\left|C_{i}\right|}$ for three different SMEFT WCs above $\sim 2 \mathrm{TeV}$. One can in principle find stronger bounds for the associated NSI coefficients in [52]. However, the range of neutrino energies used to derive these limits is mostly above the electroweak scale and therefore cannot be used in our analysis.

We compare the constraining power of neutrino NSI with that of other experimental signatures in the SMEFT. We consider the following two classes of WCs:

(i) Lepton flavor violating (LFV) coefficients: The lack of signals of charged lepton flavor violating (CLFV) processes is known to strongly constrain the parameter space of many NP scenarios. This is expected to hold also for the SMEFT. Here we consider the radiative decays $\mu \rightarrow e \gamma, \tau \rightarrow e \gamma$, and $\tau \rightarrow \mu \gamma$, explored in detail in the context of the LEFT [43]. For each of the LFV scenarios considered in our phenomenological analysis, we derive a limit on the SMEFT combination $\Lambda_{\mathrm{UV}} / \sqrt{\left|C_{i}\right|}$. This is achieved with the same method as for neutrino NSI: for several values of $\Lambda_{\mathrm{UV}}$, the RGEs are evaluated down to the electroweak scale, where the SMEFT and LEFT are matched at tree level, and then we further run down to $\Lambda_{\mathrm{IR}}$, where we impose the current 90\% C.L. bounds on the branching ratios of these processes [53-55] to determine bounds on the LEFT WCs. This indirectly translates into bounds on the SMEFT, in exactly the same way neutrino NSI experimental bounds do. This way, we obtain limits for two LFV SMEFT WCs. We also compute the
$Z \rightarrow \tau \mu$ branching ratio as a function of $C_{H_{23}}^{(1)}$ and $C_{{ }_{23}}^{(3)}$ using the effective coupling in Eq. (A8) and then compare it to the experimental limit from [56] to set a bound on the size of these WCs. In addition, we take the bounds compiled in Ref. [57] for $C_{l q}^{(1)}$, $C_{l_{q q}}^{(1)}, C_{2, q}^{(3)}, C_{\ell_{2311}}$, and $C_{2311}$. As expected, the limits on the SMEFT WCs derived from $\mu-e$ flavor violating processes are much stronger than those from $\tau-\mu$ or $\tau-e$ processes.

(ii) Lepton flavor conserving (LFC) coefficients: References $[58,59]$ compile bounds on flavor conserving four-fermion SMEFT operators derived from a plethora of low-energy experiments. The list includes lepton colliders, neutrino scattering on electron or nucleon targets, atomic parity violation, parity-violating electron scattering, as well as several precisely measured decays. For the coefficient $C_{{ }_{11}}^{(3)}$, we extract the bound from [60] and for $C_{\substack{\ell q \\ 3311}}^{(3)}$ we use LHC ditau measurements [61]. The authors of these references present their results in the form of $68 \%$ C.L. ranges for the coefficients the LFC operators. Assuming a Gaussian distribution, and taking into account some minor differences in notation and conventions, we translate these ranges into $90 \%$ C.L. bounds in order to have a fair comparison to the bounds derived from neutrino

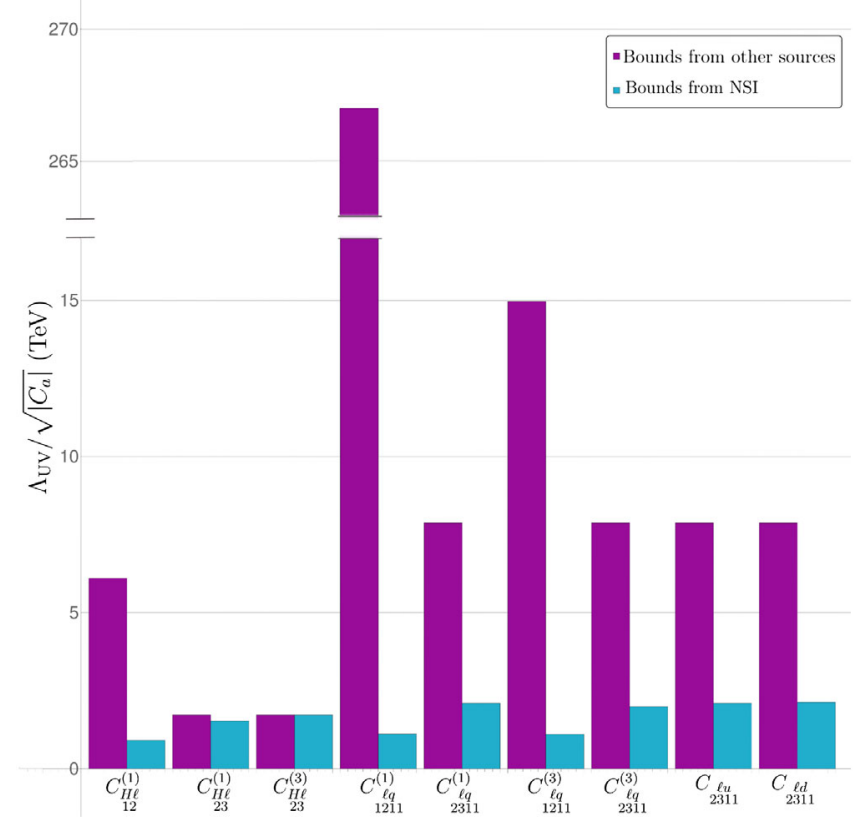

FIG. 6. Lower limits on $\Lambda_{\mathrm{UV}} / \sqrt{\left|C_{i}\right|}$, with $\Lambda_{\mathrm{UV}}$ the NP scale and $C_{i}$ the SMEFT WC, for several LFV SMEFT WCs, derived from neutrino NSI (blue bars) and from LFV processes (purple bars). See text for details. 


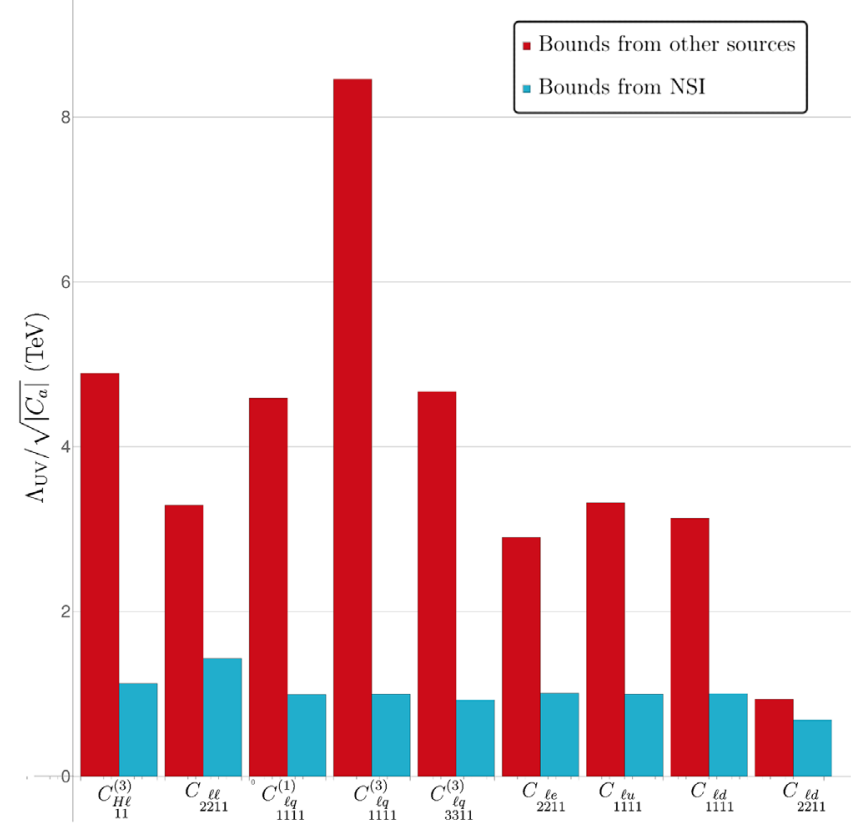

FIG. 7. Lower limits on $\Lambda_{\mathrm{UV}} / \sqrt{\left|C_{i}\right|}$, with $\Lambda_{\mathrm{UV}}$ the NP scale and $C_{i}$ the SMEFT WC, for several LFC SMEFT WCs, derived from neutrino NSI (blue bars) and from several LFC processes (red bars).
NSI. We get limits on $\Lambda_{\mathrm{UV}} / \sqrt{\left|C_{i}\right|}$ for seven LFC WCs.

Our results are presented in Figs. 6 and 7 and compiled in Table II. As anticipated, the limits from CLFV decays are more stringent than those from neutrino NSI. In fact, in some cases, the NP scale is constrained to be above $\sim 15 \mathrm{TeV}$ for $\mathcal{O}(1)$ WCs. Also, the bounds obtained from low-energy precision measurements (and extracted from $[58,59])$ are typically more constraining than those derived from neutrino NSI.

We point out that the weakest limit obtained with neutrino NSI experiments is for the $C_{2211}$ coefficient, restricted to $\Lambda_{\mathrm{UV}} / \sqrt{\left|C_{221}\right|}>690 \mathrm{GeV}$. Since $690 \mathrm{GeV}$ is well above the electroweak scale, we consider the SMEFT approach justified. In any case, the bound from low-energy precision measurements [58,59] is stronger, pushing $\Lambda_{\mathrm{UV}}$ to almost the TeV scale and, therefore, the potential NP degrees of freedom contributing to $C_{2211}$ would in principle be even heavier.

Finally, a comment on the relevance of RGE running is in order. Our previous analysis includes the full one-loop RGE running in the SMEFT and the LEFT implemented in DsixTools $[18,19]$. It is therefore essential to assess the

TABLE II. Lower limits on $\Lambda_{\mathrm{UV}} / \sqrt{\left|C_{i}\right|}$, with $\Lambda_{\mathrm{UV}}$ the NP scale and $C_{i}$ the SMEFT WC. This tables compares the limits derived from neutrino NSI with the limits obtained from other experimental signatures: LFV processes as well as collider experiments and low-energy LFC measurements.

\begin{tabular}{|c|c|c|c|c|}
\hline SMEFT & $\begin{array}{c}\text { NSI } \\
\text { coefficient }\end{array}$ & $\Lambda_{\mathrm{UV}} / \sqrt{\left|C_{i}\right|}(\mathrm{TeV})$ & Other process & $\Lambda_{\mathrm{UV}} / \sqrt{\left|C_{i}\right|}(\mathrm{TeV})$ \\
\hline$C_{H e}^{(1)}$ & $\epsilon_{e \mu}^{u L}$ & $>0.91$ & $\mu \rightarrow e \gamma$ & $>6.10$ \\
\hline$C_{H \ell}^{12}(1)$ & $\epsilon_{\mu \tau}^{d V}$ & $>1.53$ & $Z \rightarrow \mu \tau$ & $>1.72$ \\
\hline$C_{H e}^{23}$ & $\epsilon_{e e}^{e L}$ & $>1.13$ & LHC [60] & $>4.89$ \\
\hline$C_{H e}^{11}$ & $\epsilon_{\mu \tau}^{d V}$ & $>1.72$ & $Z \rightarrow \mu \tau$ & $>1.72$ \\
\hline$C_{2211}$ & $\epsilon_{\mu \mu}^{e L}$ & $>1.43$ & {$[58,59]$} & $>3.29$ \\
\hline$C_{\ell q}^{(2,1)}$ & $\epsilon_{e e}^{u V}$ & $>0.99$ & {$[58,59]$} & $>4.58$ \\
\hline$C_{e q}^{(1)}$ & $\epsilon_{e \mu}^{u L}$ & $>1.11$ & $\mu \rightarrow e$ in $\mathrm{Ti}$ [57] & $>267.06$ \\
\hline$C_{t q}^{(1)}$ & $\epsilon_{\mu \tau}^{d V}$ & $>2.10$ & $\tau$ decays [57] & $>7.87$ \\
\hline$C_{\text {tq }}^{2311}$ & $\epsilon_{e e}^{u V}$ & $>1.00$ & {$[58,59]$} & $>8.65$ \\
\hline$C_{t q}^{(3)}$ & $\epsilon_{e \mu}^{u L}$ & $>1.10$ & $\mu \rightarrow e \gamma$ & $>14.97$ \\
\hline$C_{e q}^{1211}$ & $\epsilon_{\mu \tau}^{d V}$ & $>1.99$ & $\tau$ decays [57] & $>7.87$ \\
\hline$C_{\text {eq }}^{2311}$ & $\epsilon_{\tau \tau}^{d V}$ & $>0.93$ & LHC [61] & $>4.67$ \\
\hline$C_{2.11}^{3311}$ & $\epsilon_{\mu \mu}^{e R}$ & $>1.01$ & {$[58,59]$} & $>2.90$ \\
\hline$C_{1111}$ & $\epsilon_{e e}^{u V}$ & $>1.03$ & {$[58,59]$} & $>3.32$ \\
\hline$C_{2311}$ & $\epsilon_{\mu \tau}^{u V}$ & $>2.10$ & $\tau$ decays [57] & $>7.87$ \\
\hline$C_{\ell=1}^{2311}$ & $\epsilon_{e e}^{d V}$ & $>1.01$ & {$[58,59]$} & $>3.12$ \\
\hline$C_{2 d 1} e_{221}^{111}$ & $\epsilon_{\mu \mu}^{d V}$ & $>0.69$ & {$[58,59]$} & $>0.94$ \\
\hline$C_{23 d 1}^{2211}$ & $\epsilon_{\mu \tau}^{d V}$ & $>2.13$ & $\tau$ decays [57] & $>7.87$ \\
\hline
\end{tabular}


importance of the RGE running in our procedure. To do so, we have repeated the process described in Sec. IV and computed the NSI coefficients resulting from the different SMEFT scenarios considered in our analysis, this time without RGE running. Comparing to our previous results, one can evaluate the relevance of running effects. For $\Lambda_{\mathrm{UV}}=1 \mathrm{TeV}$, the relative difference in the resulting NSI coefficients lies between 5\% and 10\%. This difference grows, as expected, for higher NP scales. One can also determine the impact on the bounds on $\Lambda_{\mathrm{UV}} / \sqrt{\left|C_{i}\right|}$. For example, in the SMEFT scenario with a nonvanishing $C_{{ }_{23}}^{(1)}$, the derived bound changes from 1.53 to $1.61 \mathrm{TeV}$. A similar change takes place in case of $C_{2 q}^{(3)}$, which goes from $1.99 \mathrm{TeV}$ when RGE running is included to $1.89 \mathrm{TeV}$ when it is absent. Therefore, although a numerical change can be noticed in some cases, the global picture would not be affected if running effects are neglected. Nevertheless, we emphasize that this conclusion holds for the scenarios considered in our exploratory analysis. One cannot discard more relevant running effects in other regions of the vast SMEFT parameter space.

\section{SUMMARY AND DISCUSSION}

Neutrino NSI constitute a powerful method to constrain $\mathrm{NP}$ at low energies. However, due to the absence of direct experimental evidence of their existence, the NP degrees of freedom might actually lie at very high energies, clearly above the electroweak scale. In this paper, we bridge the energy gap between the experiments setting limits on the neutrino NSI coefficients and the parameters of the SMEFT, an EFT valid at high energies. This connection allows for an easy application of our results to a very general class of NP models.

Our main results are shown in Figs. 6 and 7 and compiled in Table II. We conclude that current NSI limits already push the NP scale above the $\mathrm{TeV}$ in most cases. We also find that limits from other experimental probes, in particular from low-energy measurements or lepton flavor violating searches, are stronger and require higher values for $\Lambda_{\mathrm{UV}}$. While the results obtained in our analysis lead to the same qualitative conclusions reached by previous works [10-12], we emphasize the inclusion of full one-loop RGE running effects at low and high energies. This has allowed us to derive robust bounds on the SMEFT WCs and assess the numerical relevance of the running effects in the scenarios we have considered.

There are several ways in which our analysis can be extended. It is well-known that dimension-eight operators may play a relevant role; see, for instance, [10]. One should also bear in mind that our analysis assumes one SMEFT $\mathrm{WC}$ at a time. In more general scenarios, cancellations are in principle expected, potentially weakening the bounds. Similarly, we note that many of the SMEFT operators considered in our analysis generate both NC-NSI and CCNSI, and therefore these should be taken into account simultaneously in order to derive consistent constraints. Finally, one can extend the SMEFT with additional fields. For instance, operators involving light sterile neutrinos, singlet under the SM gauge group, have been considered in several works $[40,62-66]$. They allow for new scalar and tensorial neutrino four-fermion interactions at low energies $[15,16]$, recently shown to offer new phenomenological possibilities of interest $[17,36]$.

\section{ACKNOWLEDGMENTS}

The authors are grateful to Valentina De Romeri, Martin Jung, and Christoph A. Ternes for many enlightening discussions and useful comments. A. V. is grateful to Martín González-Alonso for fruitful discussions and clarifications about Refs. [58,59]. J. T.-C. is thankful to M. T. and A. V. for their great support in the beginning of his academic career. Work supported by the Spanish Grants No. FPA2017-85216-P (MINECO/AEI/FEDER, UE), No. SEJI/2018/033, and No. PROMETEO/2018/ 165 (Generalitat Valenciana), and Grant No. FPA201790566-REDC (Red Consolider MultiDark). M. T. acknowledges financial support from MINECO through the Ramón y Cajal Contract No. RYC-2013-12438.

\section{APPENDIX: SMEFT IN THE BROKEN PHASE}

Several SM parameters get modified after electroweak symmetry breaking due to contributions from dimensionsix SMEFT operators. We compile in this appendix their explicit analytical expressions. These definitions have been extracted from [9].

The Higgs vacuum expectation value is modified by the operator $Q_{H}=C_{H}\left(H^{\dagger} H\right)^{3}$, which describes a six-Higgs interaction, as

$$
v_{T} \equiv\left(1+\frac{3 C_{H} v^{2}}{8 \lambda \Lambda_{\mathrm{UV}}^{2}}\right) v
$$

We define the expansion parameter

$$
\delta_{T} \equiv \frac{v_{T}}{\Lambda_{\mathrm{UV}}}(\ll 1) .
$$

The gauge couplings, the weak mixing angle, and the effective photon and $Z$-boson couplings get also modified by dimension-six SMEFT operators involving the SM gauge fields and the Higgs doublet. They are given by

$$
\begin{aligned}
& \bar{g}_{1}=g_{1}\left(1+C_{H B} \delta_{T}^{2}\right), \quad \bar{g}_{2}=g_{2}\left(1+C_{H W} \delta_{T}^{2}\right), \\
& \cos \bar{\theta} \equiv \bar{c}=\frac{\bar{g}_{2}}{\sqrt{\bar{g}_{1}^{2}+\bar{g}_{2}^{2}}}\left[1-\delta_{T}^{2} \frac{C_{H W B}}{2} \frac{\bar{g}_{1}}{\bar{g}_{2}}\left(\frac{\bar{g}_{2}^{2}-\bar{g}_{1}^{2}}{\bar{g}_{2}^{2}+\bar{g}_{1}^{2}}\right)\right], \\
& \sin \bar{\theta} \equiv \bar{s}=\frac{\bar{g}_{1}}{\sqrt{\bar{g}_{1}^{2}+\bar{g}_{2}^{2}}}\left[1+\delta_{T}^{2} \frac{C_{H W B}}{2} \frac{\bar{g}_{2}}{\bar{g}_{1}}\left(\frac{\bar{g}_{2}^{2}-\bar{g}_{1}^{2}}{\bar{g}_{2}^{2}+\bar{g}_{1}^{2}}\right)\right]
\end{aligned}
$$


and

$$
\begin{aligned}
\bar{e} & =\bar{g}_{2} \bar{s}-\frac{1}{2} \bar{c} \bar{g}_{2} \delta_{T}^{2} C_{H W B}, \\
\bar{g}_{Z}^{2} & =\frac{\bar{e}}{\bar{s} \bar{c}}\left[1+\frac{\bar{g}_{1}^{2}+\bar{g}_{2}^{2}}{2 \bar{g}_{1} \bar{g}_{2}} \delta_{T}^{2} C_{H W B}\right] .
\end{aligned}
$$

Finally, the fermion couplings to the $W$ and $Z$ bosons are also modified by dimension-six SMEFT operators. The $W$-boson couplings are given by

$$
\begin{aligned}
{\left[W_{\ell}\right]_{p r} } & =\left[\delta_{p r}+\delta_{T}^{2} C_{p \ell}^{(3)}\right], \\
{\left[W_{R}\right]_{p r} } & =\left[\frac{1}{2} \delta_{T}^{2} C_{H_{p r}}\right],
\end{aligned}
$$

while the Z-boson couplings can be expressed as

$$
\left[Z_{\nu}\right]_{p r}=\left[\frac{1}{2} \delta_{p r}-\frac{1}{2} \delta_{T}^{2} C_{p l}^{(1)}+\frac{1}{2} \delta_{T}^{2} C_{p l}^{(3)}\right],
$$

$$
\begin{aligned}
& {\left[Z_{e_{L}}\right]_{p r}=\left[\delta_{p r}\left(-\frac{1}{2}+\bar{s}^{2}\right)-\frac{1}{2} \delta_{T}^{2} C_{p r}^{(1)}-\frac{1}{2} \delta_{T}^{2} C_{p r}^{(3)}\right],} \\
& {\left[Z_{e_{R}}\right]_{p r}=\left[\delta_{p r} \bar{s}^{2}-\frac{1}{2} \delta_{T}^{2} C_{p r}\right],} \\
& {\left[Z_{u_{L}}\right]_{p r}=\left[\delta_{p r}\left(\frac{1}{2}-\frac{2}{3} \bar{s}^{2}\right)-\frac{1}{2} \delta_{T}^{2} C_{{ }_{p r}}^{(1)}+\frac{1}{2} \delta_{T}^{2} C_{p r}^{(3)}\right],} \\
& {\left[Z_{u_{R}}\right]_{p r}=\left[\delta_{p r}\left(-\frac{2}{3} \bar{s}^{2}\right)-\frac{1}{2} \delta_{T}^{2} C_{p_{p r}}\right],} \\
& {\left[Z_{d_{L}}\right]_{p r}=\left[\delta_{p r}\left(-\frac{1}{2}+\frac{1}{3} \bar{s}^{2}\right)-\frac{1}{2} \delta_{T}^{2} C_{{ }_{p r}}^{(1)}-\frac{1}{2} \delta_{T}^{2} C_{{ }_{p r}}^{(3)}\right],} \\
& {\left[Z_{d_{R}}\right]_{p r}=\left[\delta_{p r} \frac{1}{3} \bar{s}^{2}-\frac{1}{2} \delta_{T}^{2} C_{{ }_{p r}}\right] .}
\end{aligned}
$$

[1] P. F. de Salas, D. V. Forero, C. A. Ternes, M. Tortola, and J. W. F. Valle, Status of neutrino oscillations 2018: $3 \sigma$ hint for normal mass ordering and improved $C P$ sensitivity, Phys. Lett. B 782, 633 (2018).

[2] L. Wolfenstein, Neutrino oscillations in matter, Phys. Rev. D 17, 2369 (1978).

[3] J. W. F. Valle, Resonant oscillations of massless neutrinos in matter, Phys. Lett. B 199, 432 (1987).

[4] E. Roulet, MSW effect with flavor changing neutrino interactions, Phys. Rev. D 44, R935 (1991).

[5] M. M. Guzzo, A. Masiero, and S. T. Petcov, On the MSW effect with massless neutrinos and no mixing in the vacuum, Phys. Lett. B 260, 154 (1991).

[6] Y. Farzan and M. Tortola, Neutrino oscillations and nonstandard interactions, Front. Phys. 6, 10 (2018).

[7] W. Buchmuller and D. Wyler, Effective Lagrangian analysis of new interactions and flavor conservation, Nucl. Phys. B268, 621 (1986).

[8] B. Grzadkowski, M. Iskrzynski, M. Misiak, and J. Rosiek, Dimension-six terms in the Standard Model Lagrangian, J. High Energy Phys. 10 (2010) 085.

[9] E. E. Jenkins, A. V. Manohar, and P. Stoffer, Lowenergy effective field theory below the electroweak scale: Operators and matching, J. High Energy Phys. 03 (2018) 016.

[10] W. Altmannshofer, M. Tammaro, and J. Zupan, Nonstandard neutrino interactions and low energy experiments, J. High Energy Phys. 09 (2019) 083.

[11] A. Falkowski, M. González-Alonso, and Z. Tabrizi, Reactor neutrino oscillations as constraints on effective field theory, J. High Energy Phys. 05 (2019) 173.
[12] S. Davidson and M. Gorbahn, Charged lepton flavour change and non-standard neutrino interactions, Phys. Rev. D 101, 015010 (2020).

[13] P. D. Bolton and F. F. Deppisch, Probing nonstandard lepton number violating interactions in neutrino oscillations, Phys. Rev. D 99, 115011 (2019).

[14] A. Falkowski, M. González-Alonso, and Z. Tabrizi, Consistent QFT description of non-standard neutrino interactions, arXiv:1910.02971.

[15] M. Lindner, W. Rodejohann, and X.-J. Xu, Coherent neutrino-nucleus scattering and new neutrino interactions, J. High Energy Phys. 03 (2017) 097.

[16] D. Aristizabal Sierra, V. De Romeri, and N. Rojas, COHERENT analysis of neutrino generalized interactions, Phys. Rev. D 98, 075018 (2018).

[17] I. Bischer and W. Rodejohann, General neutrino interactions from an effective field theory perspective, Nucl. Phys. B947, 114746 (2019).

[18] A. Celis, J. Fuentes-Martin, A. Vicente, and J. Virto, DsixTools: The Standard Model effective field theory toolkit, Eur. Phys. J. C 77, 405 (2017).

[19] A. Celis, J. Fuentes-Martin, P. Ruiz-Femenia, A. Vicente, and J. Virto, DsixTools 2.0, to be published.

[20] J. Kopp, M. Lindner, T. Ota, and J. Sato, Non-standard neutrino interactions in reactor and superbeam experiments, Phys. Rev. D 77, 013007 (2008).

[21] S. K. Agarwalla, P. Bagchi, D. V. Forero, and M. Tórtola, Probing non-standard interactions at Daya Bay, J. High Energy Phys. 07 (2015) 060.

[22] I. Girardi, D. Meloni, and S. T. Petcov, The Daya Bay and T2K results on $\sin ^{2} 2 \theta_{13}$ and non-standard neutrino interactions, Nucl. Phys. B886, 31 (2014). 
[23] P. S. Bhupal Dev et al., Neutrino non-standard interactions: A status report, SciPost Phys. Proc. 2, 001 (2019).

[24] O. G. Miranda and H. Nunokawa, Non standard neutrino interactions: Current status and future prospects, New J. Phys. 17, 095002 (2015).

[25] T. Ohlsson, Status of non-standard neutrino interactions, Rep. Prog. Phys. 76, 044201 (2013).

[26] O. G. Miranda, M. A. Tortola, and J. W. F. Valle, Are solar neutrino oscillations robust?, J. High Energy Phys. 10 (2006) 008.

[27] F. J. Escrihuela, O. G. Miranda, M. A. Tortola, and J. W. F. Valle, Constraining nonstandard neutrino-quark interactions with solar, reactor and accelerator data, Phys. Rev. D 80 (2009) 105009; Phys. Rev. D 80, 129908 (2009).

[28] P. Coloma, Non-standard interactions in propagation at the deep underground neutrino experiment, J. High Energy Phys. 03 (2016) 016.

[29] M. Masud and P. Mehta, Nonstandard interactions and resolving the ordering of neutrino masses at DUNE and other long baseline experiments, Phys. Rev. D 94, 053007 (2016).

[30] I. Esteban, M. C. Gonzalez-Garcia, M. Maltoni, I. MartinezSoler, and J. Salvado, Updated constraints on non-standard interactions from global analysis of oscillation data, J. High Energy Phys. 08 (2018) 180.

[31] M. G. Aartsen et al. (IceCube Collaboration), Search for nonstandard neutrino interactions with IceCube DeepCore, Phys. Rev. D 97, 072009 (2018).

[32] Y. Farzan, A model for large non-standard interactions of neutrinos leading to the LMA-dark solution, Phys. Lett. B 748, 311 (2015).

[33] Y. Farzan and I. M. Shoemaker, Lepton flavor violating nonstandard interactions via light mediators, J. High Energy Phys. 07 (2016) 033.

[34] Y. Farzan and J. Heeck, Neutrinophilic nonstandard interactions, Phys. Rev. D 94, 053010 (2016).

[35] M. B. Gavela, D. Hernandez, T. Ota, and W. Winter, Large gauge invariant non-standard neutrino interactions, Phys. Rev. D 79, 013007 (2009).

[36] I. Bischer and W. Rodejohann, General neutrino interactions at the DUNE near detector, Phys. Rev. D 99, 036006 (2019).

[37] E. E. Jenkins, A. V. Manohar, and M. Trott, Renormalization group evolution of the Standard Model dimension six operators I: Formalism and lambda dependence, J. High Energy Phys. 10 (2013) 087.

[38] E. E. Jenkins, A. V. Manohar, and M. Trott, Renormalization group evolution of the Standard Model dimension six operators II: Yukawa dependence, J. High Energy Phys. 01 (2014) 035.

[39] R. Alonso, E. E. Jenkins, A. V. Manohar, and M. Trott, Renormalization group evolution of the Standard Model dimension six operators III: Gauge coupling dependence and phenomenology, J. High Energy Phys. 04 (2014) 159.

[40] R. Alonso, H.-M. Chang, E. E. Jenkins, A. V. Manohar, and B. Shotwell, Renormalization group evolution of dimension-six baryon number violating operators, Phys. Lett. B 734, 302 (2014).

[41] W. Dekens and P. Stoffer, Low-energy effective field theory below the electroweak scale: Matching at one loop, J. High Energy Phys. 10 (2019) 197.
[42] E. E. Jenkins, A. V. Manohar, and P. Stoffer, Low-energy effective field theory below the electroweak scale: Anomalous dimensions, J. High Energy Phys. 01 (2018) 084.

[43] W. Dekens, E. E. Jenkins, A. V. Manohar, and P. Stoffer, Non-perturbative effects in $\mu \rightarrow e \gamma$, J. High Energy Phys. 01 (2019) 088.

[44] I. Brivio et al., Computing tools for the SMEFT, in Computing Tools for the SMEFT, edited by J. Aebischer, M. Fael, A. Lenz, M. Spannowsky, and J. Virto (2019), https://arxiv.org/abs/1910.11003.

[45] J. Aebischer et al., WCxf: An exchange format for Wilson coefficients beyond the Standard Model, Comput. Phys. Commun. 232, 71 (2018).

[46] F. J. Escrihuela, M. Tortola, J. W. F. Valle, and O. G. Miranda, Global constraints on muon-neutrino non-standard interactions, Phys. Rev. D 83, 093002 (2011).

[47] A. Bolanos, O. G. Miranda, A. Palazzo, M. A. Tortola, and J. W. F. Valle, Probing non-standard neutrino-electron interactions with solar and reactor neutrinos, Phys. Rev. D 79, 113012 (2009).

[48] S. Davidson, C. Pena-Garay, N. Rius, and A. Santamaria, Present and future bounds on nonstandard neutrino interactions, J. High Energy Phys. 03 (2003) 011.

[49] J. Barranco, O. G. Miranda, C. A. Moura, and J. W. F. Valle, Constraining non-standard neutrino-electron interactions, Phys. Rev. D 77, 093014 (2008).

[50] P. Coloma, M. C. Gonzalez-Garcia, M. Maltoni, and T. Schwetz, COHERENT enlightenment of the neutrino dark side, Phys. Rev. D 96, 115007 (2017).

[51] M. C. Gonzalez-Garcia, M. Maltoni, and J. Salvado, Testing matter effects in propagation of atmospheric and longbaseline neutrinos, J. High Energy Phys. 05 (2011) 075.

[52] J. Salvado, O. Mena, S. Palomares-Ruiz, and N. Rius, Nonstandard interactions with high-energy atmospheric neutrinos at IceCube, J. High Energy Phys. 01 (2017) 141.

[53] A. M. Baldini et al., MEG upgrade proposal, arXiv: 1301.7225 .

[54] A. M. Baldini et al. (MEG Collaboration), Search for the lepton flavour violating decay $\mu^{+} \rightarrow \mathrm{e}^{+} \gamma$ with the full dataset of the MEG experiment, Eur. Phys. J. C 76, 434 (2016).

[55] B. Aubert et al. (BABAR Collaboration), Searches for Lepton Flavor Violation in the Decays $\tau^{ \pm} \rightarrow e^{ \pm} \gamma$ and $\tau^{ \pm} \rightarrow \mu^{ \pm} \gamma$, Phys. Rev. Lett. 104, 021802 (2010).

[56] G. Aad et al. (ATLAS Collaboration), Search for leptonflavour-violating decays of the Higgs and $Z$ bosons with the ATLAS detector, Eur. Phys. J. C 77, 70 (2017).

[57] M. Carpentier and S. Davidson, Constraints on two-lepton, two quark operators, Eur. Phys. J. C 70, 1071 (2010).

[58] A. Falkowski and K. Mimouni, Model independent constraints on four-lepton operators, J. High Energy Phys. 02 (2016) 086.

[59] A. Falkowski, M. González-Alonso, and K. Mimouni, Compilation of low-energy constraints on 4-fermion operators in the SMEFT, J. High Energy Phys. 08 (2017) 123.

[60] J. Ellis, C. W. Murphy, V. Sanz, and T. You, Updated global SMEFT fit to Higgs, diboson and electroweak data, J. High Energy Phys. 06 (2018) 146.

[61] A. Cerri et al., Report from Working Group 4, CERN Yellow Rep. Monographs 7, 867 (2019). 
[62] F. del Aguila, S. Bar-Shalom, A. Soni, and J. Wudka, Heavy Majorana neutrinos in the effective Lagrangian description: Application to Hadron colliders, Phys. Lett. B 670, 399 (2009).

[63] A. Aparici, K. Kim, A. Santamaria, and J. Wudka, Righthanded neutrino magnetic moments, Phys. Rev. D 80, 013010 (2009).

[64] S. Bhattacharya and J. Wudka, Dimension-seven operators in the standard model with right handed neutrinos, Phys.
Rev. D 94 (2016) 055022; Phys. Rev. D 95, 039904 (2017).

[65] Y. Liao and X.-D. Ma, Operators up to dimension seven in Standard Model effective field theory extended with sterile neutrinos, Phys. Rev. D 96, 015012 (2017).

[66] J. Alcaide, S. Banerjee, M. Chala, and A. Titov, Probes of the Standard Model effective field theory extended with a right-handed neutrino, J. High Energy Phys. 08 (2019) 031. 